\title{
A New Randomized Pólya Urn Model
}

\author{
Djilali Ait Aoudia, François Perron* \\ Département de Mathématiques et de Statistique, Université de Montréal, Montréal, Canada

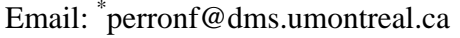

Received July 5, 2012; revised August 5, 2012; accepted August 13, 2012

\begin{abstract}
In this paper, we propose a new class of discrete time stochastic processes generated by a two-color generalized Pólya urn, that is reinforced every time. A single urn contains $a$ white balls, $b$ black balls and evolves as follows: at discrete times $n=1,2, \cdots$, we sample $M_{n}$ balls and note their colors, say $R_{n}$ are white and $M_{n}-R_{n}$ are black. We return the drawn balls in the urn. Moreover, $N_{n} R_{n}$ new white balls and $N_{n}\left(M_{n}-R_{n}\right)$ new black balls are added in the urn. The numbers $M_{n}$ and $N_{n}$ are random variables. We show that the proportions of white balls forms a bounded martingale sequence which converges almost surely. Necessary and sufficient conditions for the limit to concentrate on the set $\{0,1\}$ are given.
\end{abstract}

Keywords: Urn Model; Martingale; Asymptotic Exchangeability

\section{Introduction}

Urn models have been among the most popular probabilistic schemes and have received a lot of attention in the literature (see [1,2]). Let us describe the Pólya urn scheme briefly. In 1923, [3] proposed the following urn scheme to model processes such as the spread of infectious diseases. In this scheme, a single urn contains $a>0$ white balls and $b>0$ black balls. One ball is drawn at random and then replaced, together with $c>0$ balls of the same color. The procedure is repeated $n$ times. It is known that the sequence of the proportions of white balls is a martingale converging almost surely to a random variable having a beta distribution with parameters $a / c$ and $b / c$. Since then, numerous generalizations and extentions of the Pólya urn have been studied : see [4-14]. In 1990, [6] generalized the Pólya urn model with the single change that the number of extra balls added in the urn is a function of time. One ball is drawn and is replaced in the urn along with $F(n)$ balls of the same color. In his setup $F: \mathbb{N}^{*} \rightarrow[0,+\infty)$ can be any function. He showed that the proportions of white balls converge almost surely and the limit has no atom except possibly at 0 or 1 .

In this paper, we propose a new class of discrete time stochastic processes generated by a two-color generalized Pólya urn that is reinforced every time. This is a generalization of the urn model considered in [10]. A single urn contains $a>0$ white balls and $b>0$ black

*Corresponding author. balls: at discrete times $n=1,2, \cdots$, we draw $M_{n}$ balls and note their colors, say $R_{n}$ are black and $M_{n}-R_{n}$ are white. We return the drawn balls to the urn. Moreover, $N_{n} R_{n}$ new white balls and $N_{n}\left(M_{n}-R_{n}\right)$ new black balls are added in the urn. The numbers $M_{n}$ and $N_{n}$ are random variables in $\mathbb{N}$. We show that the proportions of white balls form a bounded martingale sequence which converges almost surely. Necessary and sufficient conditions for the limit to have no atoms at 0 or 1 are given.

This paper is organized as follows. In Section 2, we set the probabilistic model for a randomly reinforced urn. In Section 3, we show that the proportions of white balls form a bounded martingale sequence which converges almost surely. Necessary and sufficient conditions for the limit to concentrate on the set $\{0,1\}$ are given. We conclude the paper by proving that interacting reinforced urn process are asymptotically exchangeable.

\section{Model Description and Notation}

On a rich enough probability space $(\Omega, \mathfrak{I}, \mathbb{P})$, define two sequences $\left\{M_{n}\right\}$ and $\left\{N_{n}\right\}$ of positive, integervalued random variables and let $a$ and $b$ are fixed positive integers. A randomly reinforced urn generates the stochastic processes $\left\{W_{n}\right\},\left\{B_{n}\right\},\left\{R_{n}\right\}$. The sequences $B_{n}$ and $W_{n}$ respectively denote the number of black balls and white balls in the urn at time $n$. The dynamics of the processes $\left\{W_{n}\right\},\left\{B_{n}\right\}$ and $\left\{R_{n}\right\}$ are governed by the following: set $W_{0}=a, B_{0}=b$ and let 
$R_{1}$ be a Hypergeometric $\left(M_{1}, W_{0}, B_{0}\right)$ random variable. Now we iterate this sampling scheme forever. Thus, at time $n+1$, given the sigma-field $\mathfrak{I}_{n}$ generated by $R_{1}, \cdots, R_{n}, M_{1}, \cdots, M_{n+1}, N_{1}, \cdots, N_{n}$, we consider that $R_{n+1}$ is a Hypergeometric $\left(M_{n+1}, W_{n}, B_{n}\right)$ random variable and we assume that $R_{n+1}$ and $N_{n+1}$ are independent conditionaly on $\mathfrak{I}_{n}$. Finally, we set

$$
\left\{\begin{array}{l}
W_{n+1}=W_{n}+N_{n+1} R_{n+1} \\
B_{n+1}=B_{n}+N_{n+1}\left(M_{n+1}-R_{n+1}\right) \\
Z_{n+1}=\frac{W_{n+1}}{W_{n+1}+B_{n+1}}
\end{array}\right.
$$

This is a generalization of the reinforced urn model considered in [10]. Assume that, at each time periode $n=1,2, \cdots$, a new firm appears on the market and have to choose operative systems among the systems $A$ and $B$, for its $N_{n} M_{n}$ computers. The firm can choose $R_{n}$ blocks of size $N_{n}$ of computers having the operative system $A$. This choice depends on the number of computers having the operative system $A$ on the market. The process $\left\{Z_{n}\right\}$ given in (2.1) is going to describe the evolution along time of the proportion of computers operating systems $A$.

\section{Martingale Property}

The process $\left\{Z_{n}: n \geq 0\right\}$ is of primary interest for studying the stochastic processes generated by this generalization of Pólya's urn. Here $Z_{n}$ represents the proportion of white balls in the urn at time $n$. We show that these proportions form a bounded martingale. This martingale converges almost surely. The next theorem is of fundamental importance, this is our main result.

Theorem 3.1. The sequence $\left\{Z_{n}: n \geq 0\right\}$ is a bounded martingale with respect to the filtration $\mathfrak{J}_{n}$ taking values in $[0,1]$. Therefore, it converges almost surely to a random variable $Z_{\infty} \in[0,1]$.

\section{Proof.}

Here $Z_{n}=W_{n} / \alpha_{n}$ with $\alpha_{n}=a+b+\sum_{j=1}^{n} M_{j} N_{j}$ being the total number of balls in the urn at time $n$. Now let us show that $\left\{Z_{n}\right\}$ is a martingale. In fact

$$
\begin{aligned}
\mathbb{E}\left[Z_{n+1} \mid \mathfrak{I}_{n}\right] & =\mathbb{E}\left[\frac{W_{n+1} \mid \mathfrak{I}_{n+1}}{\alpha_{n}}\right]=\mathbb{E}\left[\frac{W_{n}+N_{n+1} R_{n+1}}{\alpha_{n+1}} \mid \mathfrak{I}_{n}\right] \\
& =\mathbb{E}\left[\frac{W_{n}+N_{n+1} M_{n+1} Z_{n}}{\alpha_{n+1}} \mid \mathfrak{J}_{n}\right] \\
& =\mathbb{E}\left[\frac{\alpha_{n} Z_{n}+N_{n+1} M_{n+1} Z_{n}}{\alpha_{n+1}} \mid \mathfrak{I}_{n}\right]=Z_{n} .
\end{aligned}
$$

This shows that $\left\{Z_{n}\right\}$ is $[0,1]$-bounded martingale with respect to the filtration $\mathfrak{J}_{n}$. Hence, there exists a random variable $Z_{\infty} \in[0,1]$ such that $\lim _{n \rightarrow \infty} Z_{n}=Z_{\infty}$ on a set of probability one.

The exact distribution of $Z_{\infty}$ is unknown except in a few particular cases. The situation where $M_{n}=1$ almost surely for all $n$ and $N_{n}=c>0$ almost surely for all $n$ corresponds to the classical Pólya-Eggenberger contagious urn scheme. In this case $Z_{\infty}$ has a beta distribution with parameters $a / c$ and $b / c$. Let us continue with the general case.

Theorem 3.2. The limit $Z_{\infty}$ is Bernoulli distributed if and only if

$$
\begin{aligned}
& \sum_{n=0}^{\infty} \delta_{n}=\frac{a b}{(a+b)^{2}} \\
& \text { with } \delta_{n}=\mathbb{E}\left[\frac{N_{n+1}^{2} M_{n+1} Z_{n}\left(1-Z_{n}\right)\left(\alpha_{n}-M_{n+1}\right)}{\alpha_{n+1}^{2}\left(\alpha_{n}-1\right)}\right]
\end{aligned}
$$

Proof. From (2.1) we have

$$
\begin{aligned}
& \mathbb{E}\left[\left(W_{n+1}-W_{n}\right)^{2} \mid \mathfrak{I}_{n}\right]=\mathbb{E}\left[N_{n+1}^{2} R_{n+1}^{2} \mid \mathfrak{I}_{n}\right] \\
= & \mathbb{E}\left[N_{n+1}^{2} \mid \mathfrak{I}_{n}\right]\left(\operatorname{Var}\left[R_{n+1} \mid \mathfrak{I}_{n}\right]+\mathbb{E}^{2}\left[R_{n+1} \mid \mathfrak{I}_{n}\right]\right) \\
= & \mathbb{E}\left[\frac{\left.N_{n+1}^{2} M_{n+1} Z_{n}\left(1-Z_{n}\right)\left(\alpha_{n}-M_{n+1}\right) \mid \mathfrak{I}_{n}\right]}{\alpha_{n}-1} \mid\right. \\
& +\mathbb{E}\left[N_{n+1}^{2} M_{n+1}^{2} Z_{n}^{2} \mid \mathfrak{I}_{n}\right],
\end{aligned}
$$

thus we get

$$
\begin{aligned}
& \mathbb{E}\left[Z_{n+1}^{2} \mid \mathfrak{I}_{n}\right]=\mathbb{E}\left[\frac{\left(W_{n+1}-W_{n}+W_{n}\right)^{2}}{\alpha_{n+1}^{2}} \mid \mathfrak{I}_{n}\right] \\
= & \mathbb{E}\left[\frac{\left(W_{n+1}-W_{n}\right)^{2}}{\alpha_{n+1}^{2}} \mid \mathfrak{J}_{n}\right]+\mathbb{E}\left[\frac{2 W_{n}}{\alpha_{n+1}^{2}}\left(W_{n+1}-W_{n}\right) \mid \mathfrak{J}_{n}\right] \\
& +\mathbb{E}\left[\frac{W_{n}^{2}}{\alpha_{n+1}^{2}} \mid \mathfrak{J}_{n}\right] \\
= & \mathbb{E}\left[\frac{N_{n+1}^{2} M_{n+1} Z_{n}\left(1-Z_{n}\right)\left(\alpha_{n}-M_{n+1}\right)}{\alpha_{n+1}^{2}\left(\alpha_{n}-1\right)} \mid \mathfrak{I}_{n}\right] \\
& +\mathbb{E}\left[\frac{N_{n+1}^{2} M_{n+1}^{2} Z_{n}^{2}}{\alpha_{n+1}^{2}} \mid \mathfrak{J}_{n}\right] \\
& +\mathbb{E}\left[\frac{2 \alpha_{n} Z_{n}}{\alpha_{n+1}^{2}} N_{n+1} M_{n+1} Z_{n} \mid \mathfrak{J}_{n}\right]+\mathbb{E}_{n}\left[\frac{\alpha_{n}^{2} Z_{n}^{2}}{\alpha_{n+1}^{2}} \mid \mathfrak{J}_{n}\right] \\
= & \mathbb{E}\left[\frac{N_{n+1}^{2} M_{n+1} Z_{n}\left(1-Z_{n}\right)\left(\alpha_{n}-M_{n+1}\right)}{\alpha_{n+1}^{2}\left(\alpha_{n}-1\right)} \mid \mathfrak{I}_{n}\right]+Z_{n}^{2} .
\end{aligned}
$$

The transition between the second equality and the third equality relies on the fact that, conditionally on $\mathfrak{J}_{n}, N_{n+1}$ and $R_{n+1}$ are independent. As a result 


$$
\mathbb{E}\left[Z_{n+1}^{2}\right]=\delta_{n}+\mathbb{E}\left[Z_{n}^{2}\right],
$$

with $\delta_{n}=\mathbb{E}\left[\frac{N_{n+1}^{2} M_{n+1} Z_{n}\left(1-Z_{n}\right)\left(\alpha_{n}-M_{n+1}\right)}{\alpha_{n+1}^{2}\left(\alpha_{n}-1\right)}\right]$.

Now we set $V_{n}=\mathbb{E}\left[Z_{n}^{2}\right]-a /(a+b)$, and we have from (3.1)

$$
\begin{aligned}
V_{n+1} & =\mathbb{E}\left[Z_{n+1}^{2}\right]-(a /(a+b)) \\
& =V_{n}+\delta_{n}=V_{0}+\sum_{j=0}^{n} \delta_{j}=-\frac{a b}{(a+b)^{2}}+\sum_{j=0}^{n} \delta_{j} .
\end{aligned}
$$

Consequently, $\mathbb{E}\left[Z_{n}^{2}\right]$ converges to $a /(a+b)$ if and only if $V_{n}$ converges to 0 , which happens whenever $\sum_{j=0}^{\infty} \delta_{j}=\frac{a b}{(a+b)^{2}}$. This is the desired result because a random variable $\theta \in[0,1]$ satisfies the condition $\mathbb{E}[\theta]=\mathbb{E}\left[\theta^{2}\right]$ if and only if $\theta$ is concentrated on the set $\{0,1\}$.

This theorem applies, for example, when $M_{n}=n$ and $N_{n}=2^{n}$ almost surely. In fact from (3.1), we remark that when $M_{n}=G(n)$ and $N_{n}=F(n)$ almost surely $\left(F: \mathbb{N}^{*} \rightarrow \mathbb{N}\right.$ and $G: \mathbb{N}^{*} \rightarrow \mathbb{N}$ be any function) we have

$$
\begin{aligned}
\mathbb{E}\left[Z_{n+1}^{2}\right]= & \delta_{n}+\mathbb{E}\left[Z_{n}^{2}\right] \\
= & \frac{\left(\alpha_{n}-G(n+1)\right) F^{2}(n+1) G(n+1)}{\alpha_{n+1}^{2}\left(\alpha_{n}-1\right)} \\
& \cdot\left(\frac{a}{a+b}-\mathbb{E}\left[Z_{n}^{2}\right]\right)+\mathbb{E}\left[Z_{n}^{2}\right],
\end{aligned}
$$

and we deduce

$$
\begin{aligned}
& V_{n+1}=\mathbb{E}\left[Z_{n+1}^{2}\right]-(a /(a+b)) \\
& =V_{n}\left[1-\frac{\left(\alpha_{n}-G(n+1)\right) F^{2}(n+1) G(n+1)}{\alpha_{n+1}^{2}\left(\alpha_{n}-1\right)}\right] \\
& =V_{0} \prod_{j=0}^{n}\left(1-\frac{\left(\alpha_{j}-G(j+1)\right) F^{2}(j+1) G(j+1)}{\alpha_{j+1}^{2}\left(\alpha_{j}-1\right)}\right) \\
& =\frac{a b}{(a+b)^{2}} \prod_{j=0}^{n}\left(1-\frac{\left(\alpha_{j}-G(j+1)\right) F^{2}(j+1) G(j+1)}{\alpha_{j+1}^{2}\left(\alpha_{j}-1\right)}\right) .
\end{aligned}
$$

Consequently, $\mathbb{E}\left[Z_{n}^{2}\right]$ converges to $a /(a+b)$ if and only if $V_{n}$ converges to 0 , which happens whenever the product values

$$
\left(1-\frac{\left(\alpha_{n}-G(n+1)\right) F^{2}(n+1) G(n+1)}{\alpha_{n+1}^{2}\left(\alpha_{n}-1\right)}\right) \text { converges to }
$$

0 . This happens whenever

$$
\sum_{j=0}^{\infty} \frac{\left(\alpha_{j}-G(j+1)\right) F^{2}(j+1) G(j+1)}{\alpha_{j+1}^{2}\left(\alpha_{j}-1\right)} \text { diverges. }
$$

Moreover, if $G(n)=n$ and $F(n)=2^{n}$ then

$$
\alpha_{n}=a+b+\sum_{j=1}^{n} j 2^{j}=a+b+n 2^{n+2}-(n+1) 2^{n+1}+2,
$$

the general term of the series being proportional to $n^{-1}$. From Theorem 3.1 we deduce that,

Corollary 3.3. Assume that the sequence $\left\{M_{n}: n \geq 0\right\}$ satisfies the following conditions

$$
\begin{aligned}
& \text { (1) } M_{n} \underset{n \rightarrow \infty}{\longrightarrow} M_{\infty} \text { a.s., } \\
& \text { (2) } \mathbb{P}\left(M_{\infty}<\infty\right)=1, \\
& \text { (3) } \frac{M_{n}}{B_{n}} \underset{n \rightarrow \infty}{\longrightarrow} 0 \text { a.s., }
\end{aligned}
$$

then

and

$$
R_{n} \underset{n \rightarrow \infty}{\stackrel{\mathcal{L}}{\longrightarrow}} R_{\infty},
$$

$$
\mathcal{L}\left(R_{\infty} \mid\left(M_{\infty}, Z_{\infty}\right)\right)=\operatorname{Bin}\left(M_{\infty}, Z_{\infty}\right) .
$$

Proof. For every fixed $k=0,1, \cdots$, and for sufficiently large $n$,

$$
\mathbb{P}\left(R_{n+1} \mid \mathfrak{I}_{n}\right)=\frac{\left(\begin{array}{c}
W_{n} \\
k
\end{array}\right)\left(\begin{array}{c}
B_{n} \\
M_{\infty}-k
\end{array}\right)}{\left(\begin{array}{c}
\alpha_{n} \\
M_{\infty}
\end{array}\right)}, \text { a.s. }
$$

Then, using the well know convergence of the hypergeometric probabilities to the binomial, we have

$$
\frac{\left(\begin{array}{c}
W_{n} \\
k
\end{array}\right)\left(\begin{array}{c}
B_{n} \\
M_{\infty}-k
\end{array}\right)}{\left(\begin{array}{c}
\alpha_{n} \\
M_{\infty}
\end{array}\right)} \rightarrow\left(\begin{array}{c}
M_{\infty} \\
k
\end{array}\right) Z_{\infty}^{k}\left(1-Z_{\infty}\right)^{M_{\infty}-k}
$$

and the conclusion follows from the bounded convergence theorem.

As a consequence of Theorem (3.2), we have the following.

Corollary 3.4. Assume that the sequences $\left\{M_{n}: n \geq 0\right\}$ and $\left\{N_{n}: n \geq 0\right\}$ are bounded then $\mathbb{P}\left(Z_{\infty}=0\right)+\mathbb{P}\left(Z_{\infty}=1\right)<1$.

Proof. Since the sequences $\left\{M_{n}: n \geq 0\right\}$ and $\left\{N_{n}: n \geq 0\right\}$ are bounded by some constant $K>0$ and for all $n \geq 1, Z_{n}>0$ we obtain from (3.1) that

$$
\begin{aligned}
\mathbb{E}\left[Z_{n+1}^{2}\right] & =\delta_{n}+\mathbb{E}\left[Z_{n}^{2}\right], \\
& =\mathbb{E}\left[\frac{N_{n+1}^{2} M_{n+1} Z_{n}\left(1-Z_{n}\right)\left(\alpha_{n}-M_{n+1}\right)}{\alpha_{n+1}^{2}\left(\alpha_{n}-1\right)}\right]+\mathbb{E}\left[Z_{n}^{2}\right] \\
& \leq \frac{K^{3}}{(n+1)^{2}}\left(\frac{a}{a+b}-\mathbb{E}\left[Z_{n}^{2}\right]\right)+\mathbb{E}\left[Z_{n}^{2}\right] .
\end{aligned}
$$


Let $j_{0}$ be such that $K^{3 / 2}<j_{0} \leq n$. We obtain that

$$
\begin{aligned}
V_{n+1} & =\mathbb{E}\left[Z_{n+1}^{2}\right]-(a /(a+b)) \\
& \leq \frac{K^{3}}{(n+1)^{2}}\left(\frac{a}{a+b}-\mathbb{E}\left[Z_{n}^{2}\right]\right)+\mathbb{E}\left[Z_{n}^{2}\right]-(a /(a+b)) \\
& =V_{n}\left[1-\frac{K^{3}}{(n+1)^{2}}\right]=V_{j_{0}} \prod_{j=j_{0}}^{n}\left(1-\frac{K^{3}}{(j+1)^{2}}\right) .
\end{aligned}
$$

Now, since $\sum_{j=j_{0}}^{\infty} \frac{K^{3}}{(j+1)^{2}}<\infty$, then

$\prod_{j=j_{0}}^{\infty}\left(1-\frac{K^{3}}{(j+1)^{2}}\right)>0$ and $\mathbb{E}\left[Z_{\infty}^{2}\right]<a /(a+b)=\mathbb{E}\left[Z_{\infty}\right]$ so $\mathbb{P}\left(Z_{\infty}=0\right)+\mathbb{P}\left(Z_{\infty}=1\right)<1$.

The final result in this section shows that the law of large numbers holds for interacting reinforced urn systems.

Theorem 3.5. We have

$$
\lim _{n \rightarrow \infty} \frac{\sum_{j=1}^{n} X_{j}}{n}=Z_{\infty} \text { a.s., }
$$

with $X_{n}=R_{n+1} / M_{n+1}$.

Proof. Using Cesàro's summability result we obtain that,

$$
\frac{\sum_{i=1}^{n} Z_{i}}{n} \underset{n \rightarrow \infty}{\longrightarrow} Z_{\infty} \text { a.s. }
$$

Let $U_{n}=X_{n}-Z_{n}$. Since $\mathbb{E}\left[X_{n} \mid \mathfrak{I}_{n}\right]=Z_{n}$ then we have the following,

1) $\mathbb{E}\left[U_{n}\right]=0$, for all $n \geq 1$.

2) For all $i<j$ we have

$$
\begin{aligned}
& \operatorname{Cov}\left(U_{i}, U_{j}\right)=\mathbb{E}\left[U_{i} U_{j}\right]=\mathbb{E}\left[\left(X_{i}-Z_{i}\right)\left(X_{j}-Z_{j}\right)\right] \\
& =\mathbb{E}\left[X_{i} X_{j}\right]-E\left[X_{i} Z_{j}\right]-\mathbb{E}\left[X_{j} Z_{i}\right]+\mathbb{E}\left[Z_{i} Z_{j}\right] \\
& =\mathbb{E}\left[\mathbb{E}\left[X_{i} X_{j} \mid \mathfrak{J}_{j}\right]\right]-\mathbb{E}\left[X_{i} Z_{j}\right]-\mathbb{E}\left[\mathbb{E}\left[X_{j} Z_{i} \mid \mathfrak{J}_{j}\right]\right] \\
& +\mathbb{E}\left[Z_{i} Z_{j}\right] \\
& =\mathbb{E}\left[X_{i} Z_{j}\right]-\mathbb{E}\left[X_{i} Z_{j}\right]-\mathbb{E}\left[Z_{i} Z_{j}\right]+\mathbb{E}\left[Z_{i} Z_{j}\right]=0 .
\end{aligned}
$$

This shows that the sequence $\left\{U_{n}, n \geq 0\right\}$ is orthogonal, with zero mean.

3) For $c_{n}=1 / n$, we have

$$
\sum_{n=1}^{\infty} c_{n}^{2}(\log n)^{2} \mathbb{E}\left[U_{n}^{2}\right]=\sum_{n=1}^{\infty} \frac{(\log n)^{2} \mathbb{E}\left[U_{n}^{2}\right]}{n^{2}}<\infty .
$$

Therefore, the sequence $\left\{U_{n}\right\}$ satisfies the conditions of Hall's theorem (see [15], Theorem 2.8, p. 22), and we can conclude that the series

$$
\sum_{i=1}^{n} \frac{U_{i}}{i} \text { converges } \text { a.s. }
$$

Finally, Kronecker's lemma yields

$$
\frac{\sum_{i=1}^{n} U_{i}}{n}=\frac{\sum_{i=1}^{n}\left(X_{i}-Z_{i}\right)}{n} \underset{n \rightarrow \infty}{\longrightarrow} 0 \text { a.s. }
$$

This shows that

$$
\frac{\sum_{i=1}^{n} X_{i}}{n} \underset{n \rightarrow \infty}{\longrightarrow} Z_{\infty} \text { a.s. }
$$

which is the announced result.

\section{Asymptotic Exchangeability}

According to [16] a sequence of random variables $\left\{X_{n}, n \geq 1\right\}$ is asymptotically exchangeable if the joint distribution of the sequence $\left\{X_{j+n}, n \geq 1\right\}$ converges as $j \rightarrow+\infty$ to the distribution of some exchangeable sequence $\left\{T_{n}, n \geq 1\right\}$. In particular, we have the following lemma due to [17].

Lemma 6. (Aldous)

Consider $\left(T_{n}, n \geq 1\right)$ be an infinite exchangeable sequence directed by $\Theta$.

1) Let $\Theta_{n}$ be a regular conditional distribution for $T_{n+1}$ given $\left(T_{1}, \cdots, T_{n}\right)$.

Then

$$
\Theta_{n} \underset{n \rightarrow \infty}{\longrightarrow} \Theta \text { a.s. }
$$

2) Let $\left(Y_{n}, n \geq 1\right)$ be an infinite sequence. Let $\Theta_{n}$ be a regular conditinal distribution for $Y_{n+1}$ given $\left(Y_{1}, Y_{2}, \cdots, Y_{n}\right)$, and suppose that $\Theta_{n} \underset{n \rightarrow \infty}{\longrightarrow} \Theta$ a.s.

Then

$$
\left(Y_{j+1}, Y_{j+2}, \cdots\right) \stackrel{\mathcal{L}}{=}\left(T_{1}, T_{2}, \cdots\right) \text { as } j \rightarrow \infty .
$$

Theorem 3.1 together with Lemma yield that the sequence of random variables $\left\{R_{n}, n \geq 1\right\}$ is asymptotically exchangeable. The main result of this section is the following.

Theorem 4.1. Under the conditions of Corollary 3.3, the sequence of random variables $\left\{R_{n}, n \geq 1\right\}$ is asymptotically exchangeable.

Proof. For all $n \geq 1$, the conditional distribution of $R_{n+1}$ given $\mathfrak{J}_{n}$, is such that

$$
\mathbb{P}\left(R_{n+1}=k \mid \mathfrak{I}_{n}\right)=\left(\begin{array}{c}
M_{n+1} \\
k
\end{array}\right) \frac{\left(W_{n}\right)^{\underline{k}}}{\alpha_{n}^{k}} \frac{\left(B_{n}\right)^{\frac{M_{n+1}-k}{M_{n+1}-k}}}{\alpha_{n}^{M_{n}}} \frac{\alpha^{M_{n+1}}}{\left(\alpha_{n+1}\right.} .
$$

Hence, as in Corollary 3.3, we obtain that

$$
\mathbb{P}\left(R_{n+1}=k \mid \mathfrak{I}_{n}\right) \underset{n \rightarrow \infty}{\longrightarrow}\left(\begin{array}{c}
M_{\infty} \\
k
\end{array}\right) Z_{\infty}^{k}\left(1-Z_{\infty}\right)^{M_{\infty}-k} \text { a.s. }
$$

The conclusion comes from Lemma 4.1, part (b). 


\section{Conclusions}

Urn model have been widely studied and applied in both scientific and social science disciplines. In this paper, we have proposed a general class of discrete stochastic processes generated by a two-color generalized Pólya urn. This model generalizes a model previously studied by [10]. This paper also shows that the proportion of white balls form a bounded martingale sequence which converge almost surely. Asymptotic properties and asymptotic exchangeability are given. However, the complete characterization of the limit still remains to be resolved in the future and it would be interesting to explore the possibility extensions to more than two colors.

Another important application of this urn models is to randomize treatments to patients in a clinical trial (see [18]). Consider an urn containing balls of two type, representing two treatements. Patients normally arrive sequentially, and treatment assigned on the urn composition and previous treatment outcomes. For more details analysis on this application, we refer to [13].

\section{Acknowledgements}

The authors want to thank Professor Éric Marchand for his numerous advices and helpful discussions. The second author wants to thank NSERC for the financial support.

\section{REFERENCES}

[1] H. Mahmoud, "Polya Urn Models," Chapman \& Hall/ CRC Texts in Statistical Science, 2008.

[2] N. L. Johnson and S. Kotz, "Urn Models and Their Application,” John Wiley \& Sons, New York, 1977.

[3] F. Eggenberger and G. Pólya, "Über Die Statistik Verketetter Vorgäge,” Journal of Applied Mathematics and Mechanics, Vol. 3, No. 4, 1923, pp. 279-289. doi:10.1002/zamm.19230030407

[4] B. Friedman, “A Simple Urn Model,” Communications on Pure and Applied Mathematics, Vol. 2, No. 1, 1949, pp. 59-70. doi:10.1002/cpa.3160020103

[5] B. Hill, D. Lane and W. Sudderth, "A Strong Law for Some Generalized Urn Process,” Annals of Probablity, Vol. 8, No. 2, 1980, pp. 214-226. doi:10.1214/aop/1176994772
[6] R. Pemantle, “A Time-Dependent Version of Pòlya's Urn,” Journal of Theoretical Probability, Vol. 3, No. 4, 1990, pp. 627-637. doi:10.1007/BF01046101

[7] R. Gouet, "A Martingale Approach to Strong Convergence in a Generalized Pólya-Eggenberger Urn Model," Statistics \& Probability Letters, Vol. 8, No. 3, 1993, pp. 225-228. doi:10.1016/0167-7152(89)90126-0

[8] S. Kotz, H. Mahmoud and P. Robert, "On Generalized Pólya Urn Models,” Statistics \& Probability Letters, Vol. 49, No. 2, 2000, pp. 163-173. doi:10.1016/S0167-7152(00)00045-6

[9] A. Paganoni and P. Secchi, “A Numerical Study for Comparing Two Response-Adaptive Designs for Continuous Treatment Effects," Statistical Methods and Applications, Vol. 16, No. 3, 2007, pp. 321-346. doi:10.1007/s10260-006-0042-4

[10] C. May, A. Paganoni and P. Secchi, “On a Two Color Generalized Pólya Urn,” Metron, Vol. 63, 2005, pp. 115134.

[11] M. Chen and C. Wei, “A New Urn Model," Journal of Applied Probability, Vol. 42, No. 4, 2005, pp. 964-976. doi:10.1239/jap/1134587809

[12] P. Flajolet, H. Gabbaroó and H. Pekari, “Analytic Urns,” Annals of Probability, Vol. 33, No. 3, 2005, pp. 12001233. doi:10.1214/009117905000000026

[13] P. Muliere, A. Paganoni and P. Secchi, "A Randomly Reinforced Urn,” Journal of Statistical Planning and Inference, Vol. 136, No. 6, 2006, pp. 1853-1874. doi:10.1016/j.jspi.2005.08.009

[14] C. May and N. Flournoy, "Asymptotics in ResponseAdaptive Designs Generated by a Two-Color, Randomly Reinforced Urn,” Annals of Statistics, Vol. 32, 2010, pp. 1058-1078.

[15] P. Hall and C. Heyde, "Martingale Limit Theory and Its Applications,” Academic Press, New York, 1980.

[16] J. F. C. Kingman, "Uses of Exchangeability," Annals of Probability, Vol. 6, No. 2, 1978, pp. 183-197. doi:10.1214/aop/1176995566

[17] D. Aldous, "Exchangeability and Related Topics,” École d’Été de Probabilités de Saint-Flour, XIII-1983, Lecture Notes in Math 1117, Springer, Berlin, 1985.

[18] F. Hu and W. F. Rosenberger, "The Theory of ResponseAdaptive Randomization in Clinical Trials,” John Wiley and Sons, Hoboken, 2006. 